\title{
Evidence for glide and rotation defects observed in well-ordered graphite fibers
}

\author{
M. Endo, K. Oshida, ${ }^{\text {a) }}$ K. Kobori, K. Takeuchi, and K. Takahashi \\ Faculty of Engineering, Shinshu University, 500 Wakasato, Nagano 380, Japan \\ M. S. Dresselhaus \\ Department of Electrical Engineering and Computer Science and Department of Physics, \\ Massachusetts Institute of Technology, Cambridge, Massachusetts 02139
}

(Received 28 November 1994; accepted 6 March 1995)

\begin{abstract}
New structural features observed in heat-treated vapor-grown carbon fibers (VGCF's), produced by the thermal decomposition of hydrocarbon vapor, are reported using image analysis of the lattice plane structure observed by transmission electron microscopy (TEM) and atomic force microscopy (AFM). The TEM lattice image of well-ordered graphite fibers (heat-treated VGCF's at $2800{ }^{\circ} \mathrm{C}$ ) was treated by a two-dimensional fast Fourier transform, showing sharp bright spots associated with the 002 and 100 lattice planes. The heat-treated VGCF's consist of a polygonally shaped shell, and the long and short fringe structures in the TEM lattice image reflect the 002 and 100 lattice planes, respectively. From this analysis, new facts about the lattice structure are obtained visually and quantitatively. The 002 lattice planes remain and are highly parallel to each other along the fiber axis, maintaining a uniform interlayer spacing of $3.36 \AA$. The 100 lattice planes are observed to make several inclined angles with the 002 lattice planes relative to the plane normals, caused by the gliding of adjacent graphene layers. This work visually demonstrates coexistence of the graphitic stacking, as well as the gliding of the adjacent graphene layers, with a gliding angle of about $3-20^{\circ}$. These glide planes are one of the dominant stacking defects in heat-treated VGCF's. On the other hand, turbostratic structural evidence was suggested by AFM observations. The structural model of coexisting graphitic, glide, and turbostratic structures is proposed as a transitional stage to perfect three-dimensional stacking in the graphitization process. These structural features could also occur in common carbons and in carbon nanotubes.
\end{abstract}

\section{INTRODUCTION}

The vapor-grown carbon fibers (VGCF's) in this study were obtained by hydrocarbon pyrolysis such as with benzene and with methane, using the catalytic effect of ultrafine metal particles of iron with diameters as small as $50 \AA$. The growth processes, structure, and formation mechanism of VGCF's have been characterized by a variety of techniques, such as electron microscopy, electron diffraction, and $\mathrm{x}$-ray diffraction. ${ }^{1-5}$ As-prepared vapor-grown carbon fibers have a circular cross section and show a preferred orientation in which networks of carbon planes are arranged like annular rings of a tree, and these carbon planes are parallel to one another along the fiber axis. By heat treatment of VGCF's, graphite fibers of unusually high structural order, high electrical conductivity, high bulk-modulus, and high

${ }^{a}$ Permanent address: Department of Electronics and Computer Science, Nagano National College of Technology, 713 Tokuma, Nagano 381, Japan. tensile strength have been prepared. ${ }^{2}$ The VGCF's are one of the typical graphitizing carbons, unlike pitch- and PAN-based carbon fibers. The VGCF's can be easily transformed by heat treatment at temperatures above $2500{ }^{\circ} \mathrm{C}$ into highly crystalline graphite. It has been known from $x$-ray diffraction studies ${ }^{3}$ that the VGCF's attain an almost perfect three-dimensional graphite structure when heat-treated at about $3000{ }^{\circ} \mathrm{C} .{ }^{3-6}$ The extent of the graphitization of the VGCF's is higher than that of any other known fibrous form of carbon.

Despite the extensive structural analysis of VGCF's in the past, a new aspect relating to stacking defects of the graphite crystal structure of VGCF's is reported here. The same phenomenon is found in carbon nanotubes obtained by the same process as VGCF's. The glide structure of graphite planes gives rise to a shear effect coexisting with the ideal three-dimensional graphite structure. This glide structure is observed for the first time using transmission electron microscopy (TEM) combined with an image analyzer, and is seen visually in TEM lattice fringe images as short and parallel lines 
approximately normal to the long straight 002 fringes, denoting the basal plane structure of the graphene layers. By using a computer image analysis of the TEM pictures, it is possible to obtain detailed quantitative information about this important, new aspect of the visualized lattice structure of graphite. And also by AFM observations, the presence of a glide structure coexisting with the three-dimensional graphite and turbostratic structures is indicated.

These visual structural features that are observed in well-ordered graphite fibers could occur commonly in the graphitization process, so that the proposed structural model could contribute to our understanding of the graphitizability of carbons. Furthermore, these structural concepts can also be applied to multilayered carbon nanotubes, where the numbers of carbon atoms in adjacent layers are not balanced against each other to form graphite $\mathrm{ABAB}$ stacking.

\section{EXPERIMENTAL}

A schematic diagram of the geometry of the experimental TEM and AFM observations is indicated in Fig. 1. VGCF's have a circular cross section and a preferred orientation in which the carbon networks are parallel to one another along the fiber axis. The annular ring texture orienting the central hollow core of nanometer scale diameter, which is one of the structural characteristics of the cross section of the VGCF's, is retained throughout their heat treatment. A change from a circular to a polygonal cross section (Fig. 1) is observed

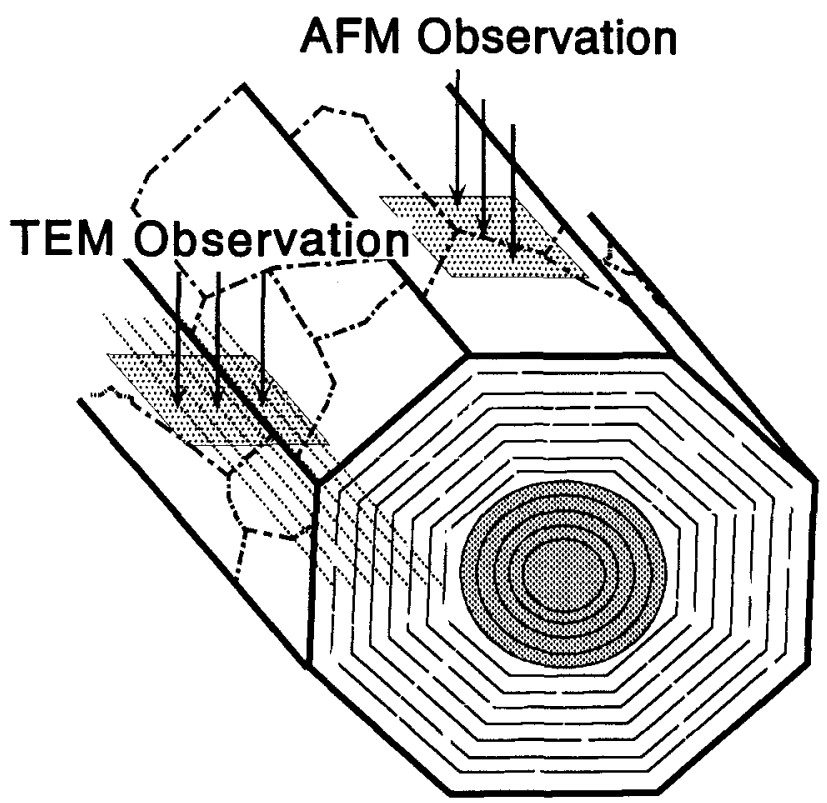

FIG. 1. Schematic diagram of the experimental arrangement for the TEM and AFM observations and a structural model for graphitized vapor-grown carbon fibers heat-treated at around $3000{ }^{\circ} \mathrm{C}$, showing the polygonal shape model for the fiber cross section. as the heat treatment temperature is increased. The fibers heat-treated above $2500{ }^{\circ} \mathrm{C}$ have a characteristic polygonal appearance in cross section, which becomes notable for heat treatment above $2800{ }^{\circ} \mathrm{C} .{ }^{7}$ For each polygonized fiber, the surfaces of the crystal grain boundaries extend predominantly along the fiber axis. By TEM and AFM observations, we can obtain the graphene layer structure along the fiber axis at the periphery of the fiber using TEM through 002 lattice fringe measurement and the atomic arrangement on the top of the fiber using AFM, respectively.

To study the lateral fringes in the lattice structure of VGCF's, it is necessary to heat-treat the as-grown VGCF's to sufficiently high temperatures as described above so that well-defined facets are formed. ${ }^{3}$ In this work, we used samples heat-treated at $2800{ }^{\circ} \mathrm{C}$ in Ar for $15 \mathrm{~min}$, so that the VGCF's became highly crystalline, like a graphite whisker, ${ }^{8}$ and the well-ordered 002 lattice planes could be observed clearly. We chose rather thin fibers (several hundred nanometers in diameter) for our TEM samples by controlling the growth conditions. The diameters of the present heat-treated fibers were less than several hundred nanometers, and 002 lattice images around the whole fiber could be observed, because of their annular ring structure.

We used an image processing system for analysis of the TEM lattice plane images, as discussed elsewhere. Figure 2(a) shows the original TEM image of the heattreated VGCF sample. This image was digitally stored in the image processing system using an image scanner, where the number of pixels is $512 \times 512$ and the density of one pixel is expressed by 8 bits (256 tones). One pixel size is $0.425 \AA$, and the digitized image size corresponds to an area of $218 \AA \times 218 \AA$. The image analysis was performed for an area of $512 \times 512$ pixels, and a part of the area, of size $400 \times 400$ pixels, is shown in Fig. 2(a). The TEM picture shows a two-dimensional projection containing the 002 direction and a direction in the basal planes perpendicular to the 002 layer planes. The long dark lines that are oriented along the fiber axis are 002 lattice planes, which have been studied extensively in the past. ${ }^{7,10,11}$ The novel features in the TEM image, which are the focus of the present study, are the short dark lines that are nearly perpendicular to the long dark 002 lines. It is shown below that these short fringes provide important new information about the stacking nature of the lattice planes present in highly graphitized VGCF's.

Image analysis has been carried out in Fig. 2(a), a typical lattice image for a heat-treated VGCF, to determine the lattice structure of VGCF's, in detail. A two-dimensional fast Fourier transform (FFT) was carried out on the original TEM image, and the power spectrum was calculated, as has been done in previous TEM studies of other systems., ${ }^{9,12}$ The resulting power spectrum is shown in Fig. 2(b). In order to suppress 


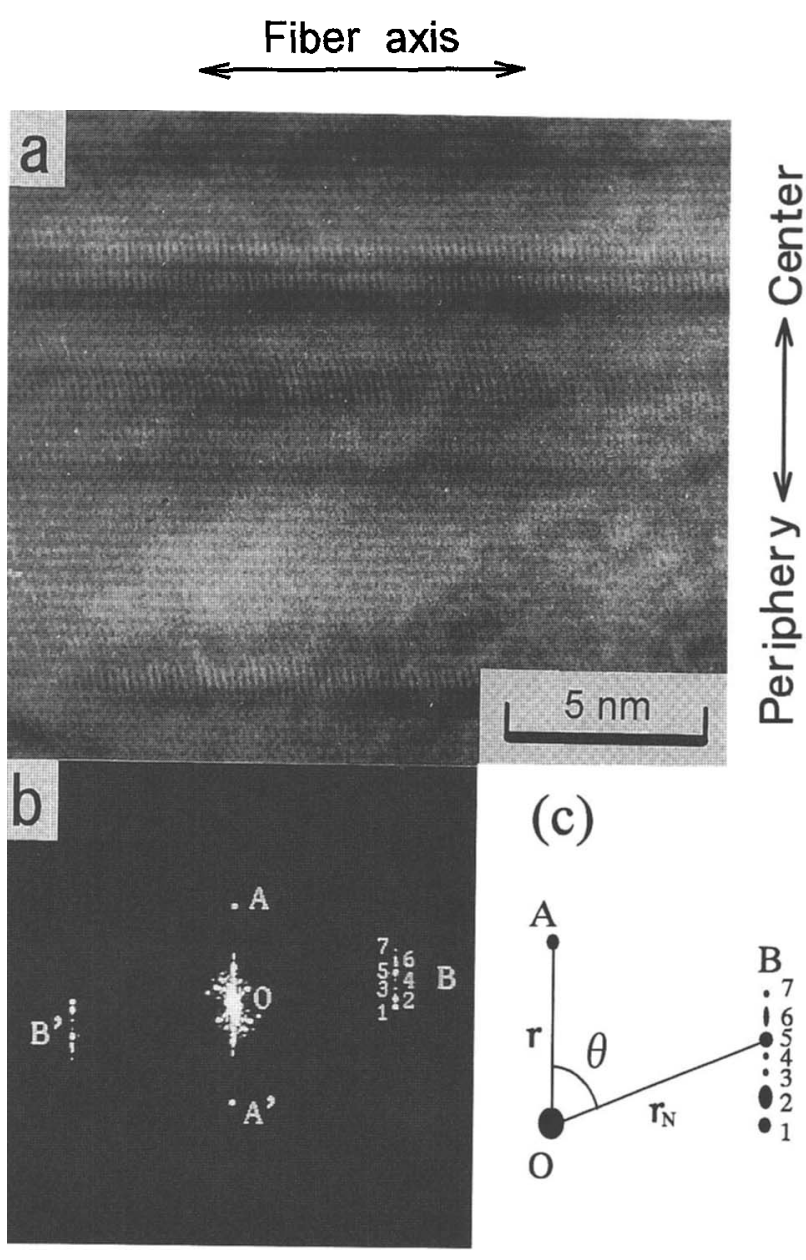

FIG. 2. (a) The digitized picture of a TEM image for a VGCF obtained from the image scanner. (b) Power spectrum of the digitized picture of the TEM image. (c) Schematic diagram showing notation used to analyze Fig. 2(b).

the influence from the peripheral regions of the original rectangular image, a Hamming window was used in preparing the FFT. Therefore, the power spectrum shown in Fig. 2(b) indicates essentially the nature of the VGCF's lattice planes.

The image after the FFT operation has the same dimensions as that of the original image $(512 \times 512$ pixels), where the real part of the FFT contains 32 bits and the imaginary part also contains 32 bits. In the power spectrum, the results from the FFT operation are exhibited by 8 bit units. The pattern of the power spectrum in Fig. 2(b) shows full inversion symmetry about the central point $O$. Thus, it is sufficient to discuss only the right-hand side of the power spectrum. The brightest spot at the center [position $O$ in Fig. 2(b)] corresponds to the dc component of the original TEM image. As the location spreads from the central point, a high frequency component appears. A detailed discussion of the power spectrum near the origin will be presented elsewhere.
Each frequency in the FFT corresponds to a periodicity found in the original image. If an inversion FFT (IFFT) of the diffraction pattern for a given frequency is carried out subsequently, an image corresponding to that frequency can be extracted. Using the power spectrum [shown in Fig. 2(b)], we selected the frequency regions of special interest by using a circular aperture. Since the real space image is reconstructed by a frequency extraction process, no window treatment, such as a Hamming window, was carried out prior to taking the FFT.

AFM observations have been carried out on a VGCF sample with a diameter of about $1 \mu \mathrm{m}$, heat-treated at the same temperature as the samples used for the TEM studies. These samples were mounted on the observation stage of the AFM using conducting carbon tape. Observations were done in air using a silicon nitride tip, and the images were treated with a low-pass filter. As a standard sample for the AFM observations, we used HOPG (highly oriented pyrolytic graphite) to calibrate the atom to atom distance.

\section{RESULTS AND DISCUSSION}

Two bright spots [positions $\mathrm{A}$ and $\mathrm{A}^{\prime}$ in Fig. 2(b)], which are symmetrically positioned about the central point $\mathrm{O}$, correspond to 002 lattice planes. Since the plots labeled $\mathrm{A}$ and $\mathrm{A}^{\prime}$ are sharp, we infer that the 002 lattice planes are highly parallel and have a uniform real space interlayer spacing.

Figure 3(b) shows the image of the IFFT formed from the FFT data included within the circles in Fig. 3(a). Related data are shown in Figs. 4 and 5. The horizontal parallel lines in Fig. 3(b) indicate 002 lattice planes associated with the spots within the circles of Fig. 3(a). Since the VGCF was heat-treated at high temperature, the repeat distances of the 002 lattice planes are calculated to be $3.36 \AA$ from the x-ray diffraction pattern, ${ }^{3}$ and this is approximately the distance between the 002 lattice planes of graphite. As seen by the sharpness of the spots in Fig. 3(a), the repeat distances of the 002 fringes are constant.

In addition, a series of spots are found in the regions labeled by $\mathrm{B}$ and $\mathrm{B}^{\prime}$ in Fig. 2(b). These spots are confined to two lines parallel to $\mathrm{AOA}^{\prime}$, on the right with spots in the upper quadrant and one on the left with spots in the lower quadrant. We relate the $\mathrm{B}$ and $\mathrm{B}^{\prime}$ spots in the diffraction pattern to the short lines in the TEM image and nearly perpendicular to the long horizontal parallel lines in Fig. 2(a). The series of spots in regions B and $\mathrm{B}^{\prime}$ indicate that the short lines in Fig. 2(a) are not parallel to each other, but make several discrete angles with the 002 lattice planes. From the multiple spots in region $B$, we select seven well-defined spots $\left[B_{1}-B_{7}\right.$, shown 


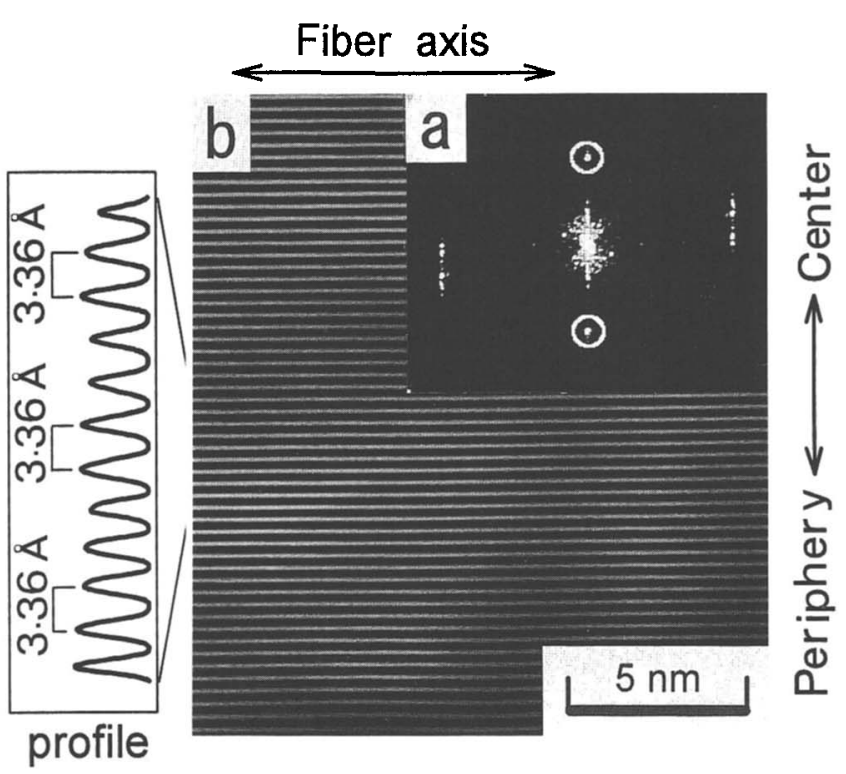

FIG. 3. The image of 002 lattice planes. (a) The power spectrum of the FFT of Fig. 2, and areas selected for the extraction of specific frequencies. (b) The image of the IFFT of areas selected in (a). The directions along the fiber axis and normal to the fiber axis are indicated; the variation in image intensity normal to the fiber axis is shown schematically on the left-hand side of the figure.

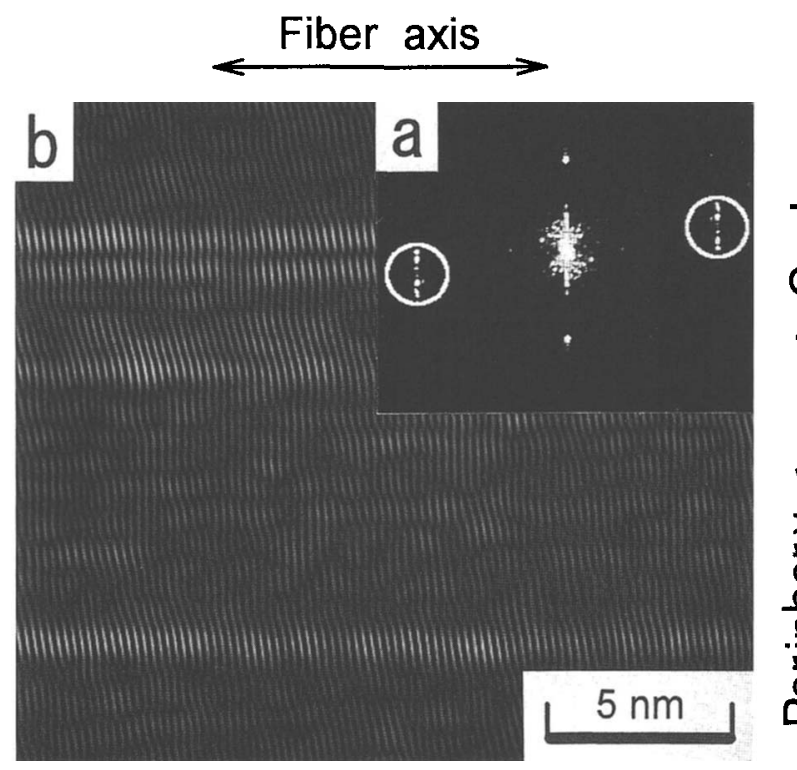

FIG. 4. The image of 100 lattice planes. (a) The power spectrum of the FFT of Fig. 2, and areas selected for the extraction of specific frequencies. (b) The image of the IFFT of areas selected in (a).

below Fig. 2(b)] for detailed analysis. The spot $\mathrm{B}_{1}$ is located on a horizontal line, which goes through the central point $\mathrm{O}$, and which is perpendicular to the $00 l$ diffraction spots on line $\mathrm{AOA}^{\prime}$. The spot $\left(\mathrm{B}_{1}\right)$ at the right thus corresponds to the 100 lattice planes. Using the distance for $d_{002}(=3.36 \AA)$ obtained from $x$-ray diffraction and the

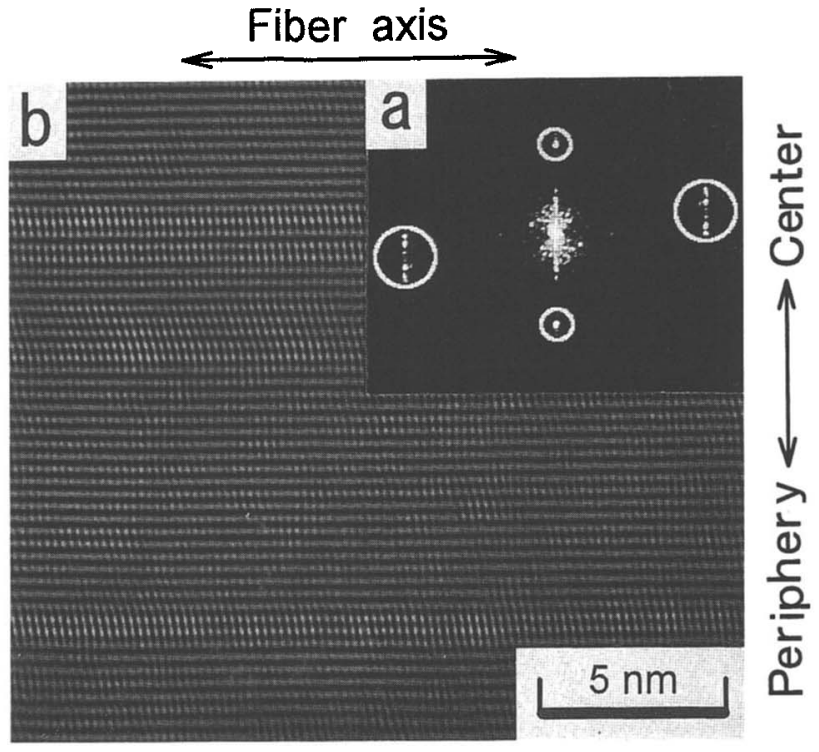

FIG. 5. The lattice image of combined 002 and 100 lattice planes. (a) The power spectrum of the FFT of Fig. 2, and areas selected for the extraction of specific lattice periodicities. (b) The image of the IFFT of areas selected in (a).

relation between spot $A$ and spot $B_{1}$, we find the spacing between the 100 lattice planes, $d_{100}$, to be $2.128 \AA$. The distance $d_{N}$ between lattice planes [see Fig. 2(c)] corresponding to the other diffraction spots $B_{2}-B_{7}$ is calculated by $d_{N}=\left(r_{1} / r_{N}\right) d_{100}$, where $r_{1}$ is the distance from the central point $O$ to the 100 spot $\left(B_{1}\right)$ in reciprocal space, and $r_{N}$ is the distance from the central point $\mathrm{O}$ to spots $\left(\mathrm{B}_{2}-\mathrm{B}_{7}\right)$. The calculated distances $d_{N}$ for lines $\mathrm{OB}_{1}-\mathrm{OB}_{7}$ are given in Table I. Since the spots $B_{2}-B_{7}$ in regions $B$ and $B^{\prime}$ are sharp, we conclude that the short lines in Fig. 2(a) correspond to parallel lattice planes with constant interlayer spacings. Since the spots $B_{2}-B_{7}$ in the region $B$ are in a row above the 100 spot, and parallel to $\mathrm{AOA}^{\prime}$ for the $00 l$ diffraction spots, we

TABLE I. Angles $\theta_{N}$ for the 100 lattice planes, which correspond to the spots $\left(B_{1}-B_{7}\right)$ in reciprocal space [shown in Fig. 2(b)], with respect to the 002 lattice planes. The interlayer distances $\left(d_{N}\right)$ between the 100 glide planes are given for each $\theta_{N}$ calculated using the 002 spacing for $N=1$ as a standard.

\begin{tabular}{lcc}
\hline \hline Spots & $\theta_{N}\left({ }^{\circ}\right)$ & $d_{N}(\AA)$ \\
\hline $\mathrm{B}_{1}$ & 90.0 & 2.13 \\
$\mathrm{~B}_{2}$ & 86.9 & 2.12 \\
$\mathrm{~B}_{3}$ & 83.2 & 2.11 \\
$\mathrm{~B}_{4}$ & 80.5 & 2.10 \\
$\mathrm{~B}_{5}$ & 77.2 & 2.08 \\
$\mathrm{~B}_{6}$ & 73.5 & 2.04 \\
$\mathrm{~B}_{7}$ & 69.9 & 2.00 \\
$\mathrm{~A}$ & $\cdots$ & 3.36 \\
$d_{002}$ determined by & $\cdots$ & 3.36 \\
$\quad$ X-ray diffraction & & \\
Graphite 101 plane & 72.4 & 2.03 \\
\hline \hline
\end{tabular}




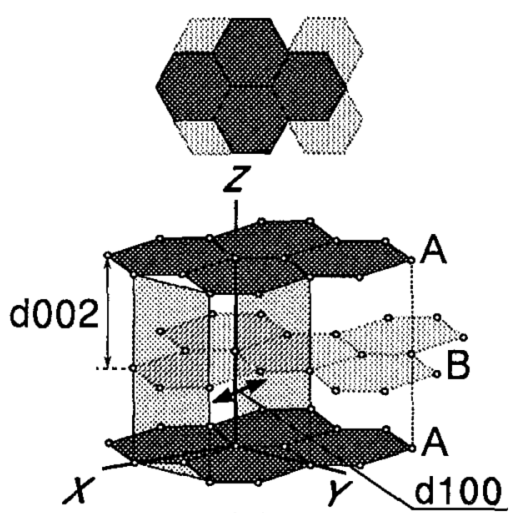

(a)

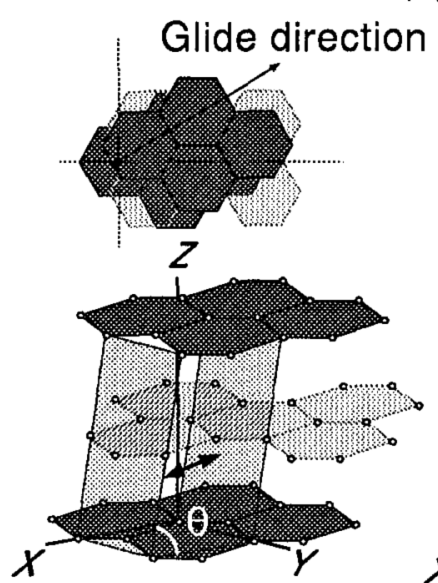

(b)

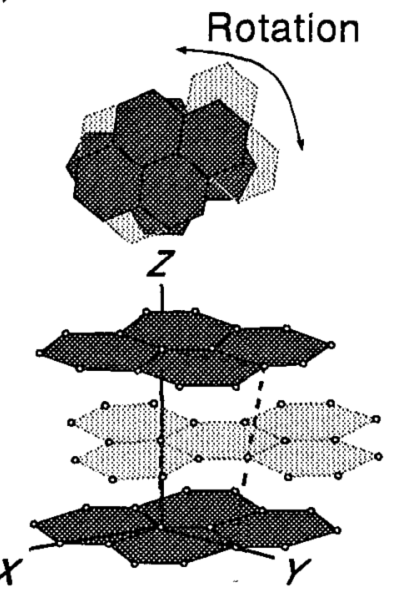

(c)
FIG. 6. Models for the lattice planes of VGCF. (a) The 100 lattice planes are perpendicular to the 002 lattice planes as in single crystal graphite, (b) glide of graphene sheets causes the 100 lattice planes to make an angle of $\theta_{N}$ with the 002 lattice planes, and (c) rotationally turbostratic graphite.

conclude that the corresponding lattice planes are tilted toward the left, while keeping their horizontal distances constant, independent of their tilt angles. The spots in region $\mathrm{B}$ thus indicate that the 002 lattice planes glide slightly from their ideal positions in the graphite lattice, as shown in Fig. 6. The angles $\theta_{N}$ for the seven spots $\mathrm{B}_{2}-\mathrm{B}_{7}$ are listed in Table $\mathrm{I}$, the angles being measured directly from the spot locations. As the angles $\theta$ between the short lines and horizontal lines become smaller, the repeat distances of the short lines become shorter. It is worth noting that the smallest angle $\theta_{7}$ (for spot $\mathrm{B}_{7}$ ) is $69.9^{\circ}$, which is smaller than the angle between the 101 lattice plane and the 002 lattice plane $\left(72.4^{\circ}\right)$, as comparatively indicated in Table I. Since the smallest angle spot $\theta_{7}$ for $B_{7}$ is located on the $B_{2}-B_{6}$ line, we may conclude that the $B_{7}$ spot could be associated with the glide series of 100 lattice planes, and not be associated with 101 planes. Fidelity in the structure could be obtained in some domains where the glide occurs in the same direction during the graphitization.
Such domains will show the three-dimensional graphite structure upon further graphitization.

To confirm the above interpretation, we extracted the spots in region $B$ and $B^{\prime}$ and carried out the IFFT. Figure 4(b) shows the image of the IFFT that includes the FFT data inside the circles in Fig. 4(a). The normal parallel lines in Fig. 4(b) correspond to 100 lattice planes. All tilted lines are rotated counterclockwise with respect to the normal parallel lines. The tilted lines further tend to be parallel to each other within regions. Although the tilt angles change continuously and gradually, there appear to be preferred tilt directions, thereby accounting for the sharp spots seen in the diffraction pattern. Figure 4(b) shows areas where the line patterns are clearly seen, and other areas where the lines are not clear, suggesting that there may be twists of the 002 lattice planes with respect to the $c$-axis. Thus the faces of the 100 lattice planes, which are not parallel to the incident electron beam of the TEM, are not seen because they do not obey the diffraction condition.

Figure 5(b) shows the image of the IFFT that includes the FFT data inside the circles in Fig. 5(a). The image shown in Fig. 5(b) clearly demonstrates that the 100 lattice planes make a variety of tilt angles with respect to the 002 lattice planes. It is important to note that the glide of the 100 planes occurs not as a mixture of random directions, but only for discrete directions having a positive angular orientation with respect to the 002 layers, as is also clear in the FFT patterns shown in Fig. 2(b). The areas where no 100 lattice fringes are observed may possibly be due to two different structures, one being out of the diffraction condition, and the other associated with turbostratic stacking. These two cases correspond to a rotational stacking model for adjacent graphene sheets, which is also commonly observed by AFM measurements, as shown below. These glide structures could be mainly associated with shear effects on the lattice planes, since facets are formed from the curved external surface during graphitization.

To explain these experimental results, we propose a model for the structure of VGCF's, as shown in Fig. 6 based on the TEM analysis. The structure of VGCF's, heat-treated at high temperature, is almost that of graphite, but with shifts of the 002 lattice planes along a basal plane direction. Figure 6(a) shows the ideal graphite structure where the 100 lattice planes are normal $\left(\theta=90^{\circ}\right)$ to the 002 lattice planes, corresponding to the spot $B_{1}$ in Fig. 2(b). When the 002 lattice planes are shifted with respect to each other as in Fig. 6(b), the 100 lattice planes make an angle $\theta<90^{\circ}$, with respect to the 002 lattice planes, and show a corresponding change in interlayer spacing. Thus, Fig. 6(b) corresponds to spots $\mathrm{B}_{2}-\mathrm{B}_{7}$ in Fig. 2(b). The measurements show that the 002 lattice planes can shift over a range of $\theta$ values, and the spot pattern in Fig. 2(b) indicates that certain shift 
angles are preferred. The ideal structure of crystalline graphite is a three-dimensional array of 002 lattice planes with $A B A B$ stacking. To explain the line of spots that is observed parallel to $\mathrm{AOA}^{\prime}$, the graphene layer $\mathrm{B}$ must shift parallel to layer A, keeping the A and B graphene planes parallel, but without the Bernal stacking shown in Fig. 6(a). Figure 6(c) shows a rotational turbostratic model, in which the upper 002 lattice planes are rotated with respect to the lower 002 lattice planes. If the two upper 002 lattice planes in Fig. 6(c) have random rotation angles with respect to each other, no diffraction pattern would be observed for the 100 lattice planes. These structures corresponding to graphitic, glide, and turbostratic stacking are located in the region where 100 lattice plane images appear and disappear, respectively. These glide structures could just be precursors of the three-dimensional graphite structure, which are formed from a turbostratic structure.

Figure 7 shows an enlarged view and a schematic representation of the mosaic structure of the stacking of graphene planes in VGCF samples. It is interesting to note that generally the graphite structure of the VGCF is sandwiched between regions showing glide stacking, and other regions showing rotational stacking. This suggests that the crystalline structure may change from turbostratic to 3-D graphite via glide plane stacking as three-dimensional graphite stacking is achieved in the graphitization process. The mosaic structure shown in Fig. 7 could minimize the energy for a structure making a transition from a turbostratic to graphite structure.

The surface layer and the underlying graphene layer of highly oriented pyrolytic graphite (HOPG) shows a hexagonal diffraction pattern with $A B A B$ stacking such that one surface atom site is directly above a carbon atom ( $\alpha$-site) in the underlying layer and the other atomic site ( $\beta$-site) is over the center of the lower hexagon. Therefore, we use HOPG as a standard comparison sample for AFM observations of a singlecrystal graphite AFM image, ${ }^{13}$ showing a trigonal lattice arrangement as indicated in Fig. 8. In this image, the atom to atom distances are exactly the same and equal to $1.42 \AA$, as indicated in the figure. The same AFM observation conditions have been selected for the VGCF samples, and typical atomic images often observed on the surface of VGCF's heat-treated at $2980{ }^{\circ} \mathrm{C}$ are shown in Fig. 9. It can be seen in this figure that some regions show a trigonal lattice, consistent with the threedimensional graphitic stacking [Fig. 9(a)], while other regions show a hexagonal image [Fig. 9(b)], indicating no evidence for three-dimensional graphitic stacking, but which corresponds to turbostratic stacking. In Fig. 9(c) we see areas of mixed trigonal and hexagonal atomic arrangements, indicating the coexistence of graphitic and disordered stacking structures. It is noteworthy that there are large regions of periodicity as seen in the

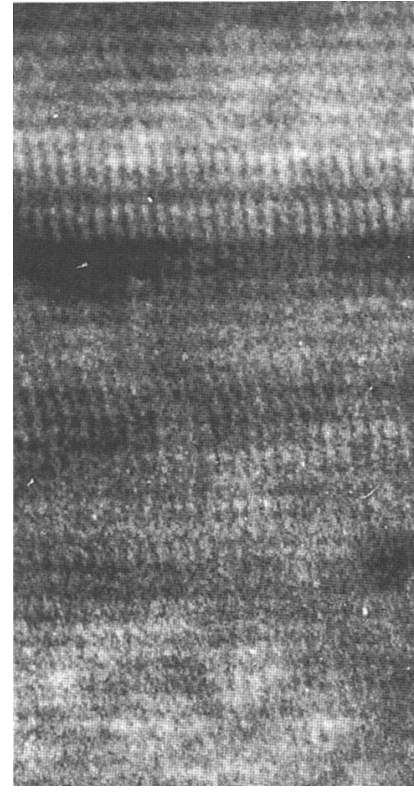

(a)

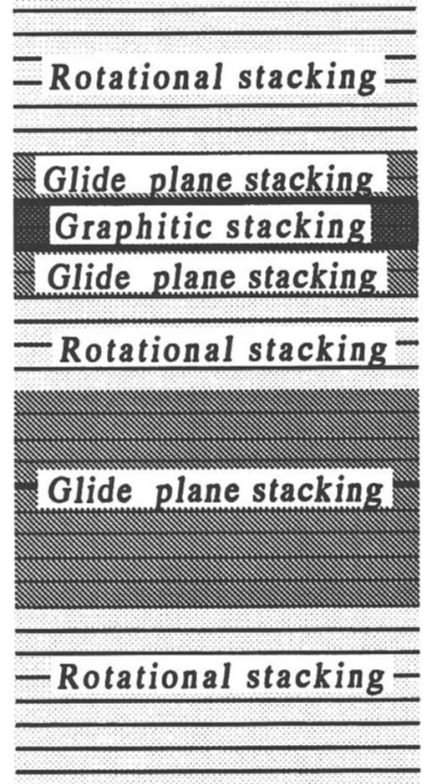

(b)

FIG. 7. The mosaic structure of heat-treated VGCF lattice planes, showing regions of graphite stacking, glide plane stacking, and rotational turbostratic stacking. (a) The TEM lattice image of VGCF. (b) A model of TEM image (a).

bright regions of the atomic image shown in Fig. 10(a). We believe that the images of Fig. 10(a) are moiré patterns due to a rotational misorientation of the two surface graphene layers resulting from the turbostratic structure. ${ }^{14}$ The periodicity $(D)$ of the moire patterns observed in Fig. 10(a) is about $1.18 \mathrm{~nm}$. The periodicity of the graphite lattice of 100 planes is $d=2.46 \AA$, and the rotation angle $\theta$ of the misorientation is given 


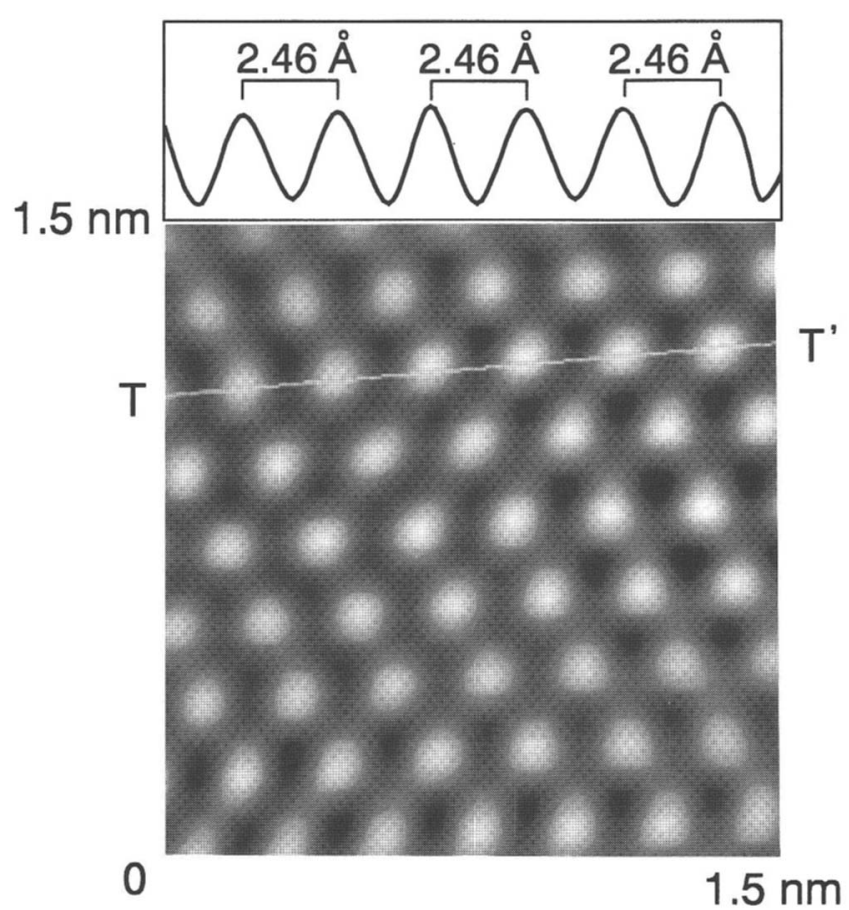

FIG. 8. An AFM image for a $1.5 \mathrm{~nm} \times 1.5 \mathrm{~nm}$ region of an $\mathrm{HOPG}$ sample showing a trigonal lattice. The intensity profile of the pattern along the $\mathbf{T}-\mathbf{T}^{\prime}$ line is shown above the AFM image.

by $\sin \theta / 2=d / 2 D$. Thus a value for $\theta$ of about $\theta=$ $12^{\circ}$ is obtained, and the corresponding atomic structure is indicated schematically in Fig. 10(b). In this model, it can be also seen that in some regions hexagons of both layers are nearly superimposed, whereas, in other regions, the stacking is similar to that of HOPG. In this image, the $\beta$-site atoms are revealed. In contrast, those areas where no moiré fringes appear, as shown in Fig. 11, could correspond to a glide structure. From the AFM observations, we conclude that VGCF's consist of large graphitic layers which are developed by heat treatment. These AFM results suggest that heat-treated VGCF's contain three stacking types of lattice planes: the first type retains the Bernal ABAB ideal graphite structure, the second is a structure containing graphene layers that glide with respect to each other, and the third is the conventional turbostratic stacking.

It is assumed that these three types of structures, including graphitic stacking, glide plane stacking, and rotational stacking, are sufficient to explain all of the structures in the observed TEM and AFM images. Thus we model graphitized VGCF's to consist of intertwined mosaic units containing three types of structures. These structures progress during the graphitization process from turbostratic stacking to glide plane stacking, and finally to the graphite structure (see Fig. 12). We further conclude that heat-treated VGCF's might require a special cylindrical structure, and this might be the reason that regions of turbostratic and glide plane structure remain through the graphitization process even at high temperatures. This model is also useful for understanding the structure of carbon nanotubes, as well as that for other carbon materials.

\section{CONCLUSION}

The microstructure of the lattice planes of VGCF's was investigated by TEM observations combined with computer image analysis, as well as AFM observations. The TEM pictures show short lines normal to the 002 lattice planes, and from analysis of the power spectrum, we conclude that these structures correspond to $100-$ related lattice planes. The 100 lattice planes within a spatial region are parallel to each other, but various regions of these types make a variety of different angles with respect to the 002 fringes corresponding to graphene layers. It is known that the 002 graphene planes slide gradually with respect to each other, as clearly seen by the FFT and IFFT results presented in this work. The angles $\theta_{N}$ between the tilted 100 lattice planes and the basal graphene planes can be measured quantitatively by image processing, and it was found that well-ordered graphite fibers contain crystallites with a structure similar to that of three-dimensional graphite, as well as imperfect crystallites showing graphene glide planes along the basal plane directions, and finally turbostratic regions are observed, where the graphene

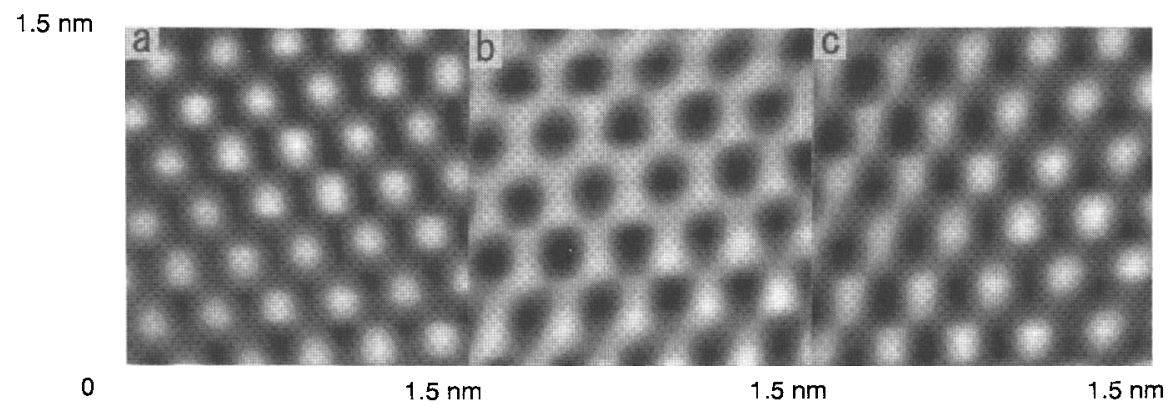

FIG. 9. (a) A trigonal AFM lattice pattern showing the three-dimensional graphitic stacking. (b) A hexagonal AFM image indicating no evidence of three-dimensional graphite stacking, and corresponding to turbostratic stacking. (c) Mixture of trigonal and hexagonal atomic arrangements, implying coexisting graphite and turbostratic structures. 


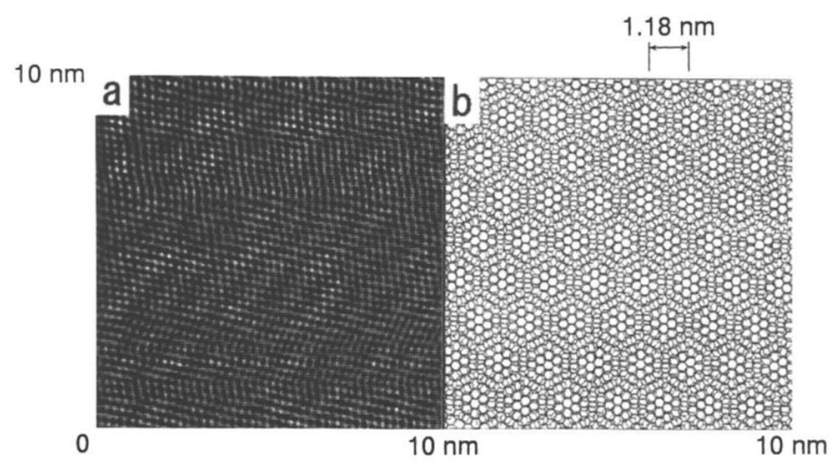

FIG. 10. Moiré patterns seen in the AFM image, and indicating a turbostratic structure as shown in (b). (b) Diagram of a moiré pattern with a rotation angle $\left(\theta=12^{\circ}\right)$ between two adjacent layers. The rotation angle is calculated by the relation $\sin \theta / 2=d / 2 D$.

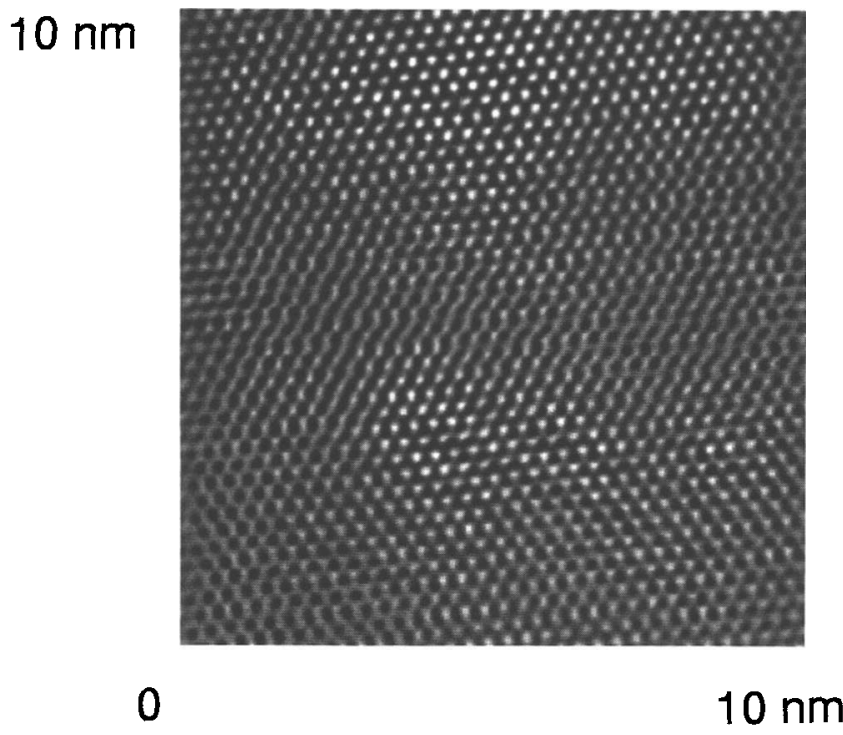

FIG. 11. An AFM image from a VGCF sample showing no evidence for moiré fringes.

planes on adjacent layers are rotated with respect to each other. The structures observed by AFM measurements provide complementary information to that from TEM measurements of structure in the $c$-direction. This work also shows that computer image processing is useful for analysis of TEM pictures to study the glide of lattice plane structures. Since the structure of VGCF's is very similar to that of carbon nanotubes, it is expected that this approach will also be useful for the analysis of the structural characteristics of carbon nanotubes, as well as for study of the graphitization behavior of various kinds of imperfectly ordered carbons.

\section{ACKNOWLEDGMENTS}

The authors wish to thank Dr. G. Dresselhaus of MIT for helpful discussions. One of the authors (ME) is indebted to Dr. A. Oberlin of the Laboratoire Marcel

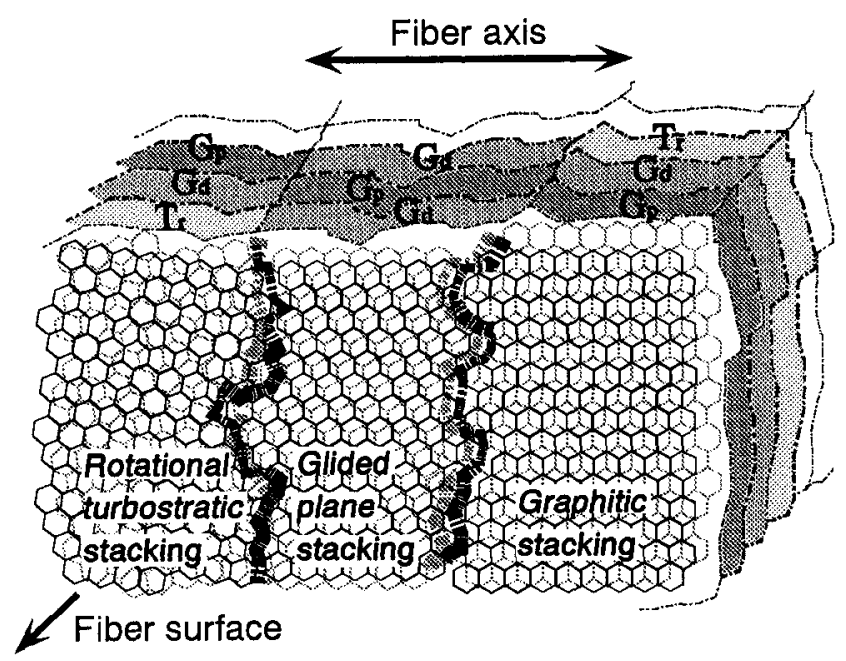

FIG. 12. In the fiber, rotational turbostratic stacking ( $\mathrm{Tr}$ ), three-dimensional graphitic stacking (Gp), and glide plane stacking (Gd) structures coexist, forming a transitional state leading to the perfect graphite crystalline structure.

Mathieu (CNRS) for useful discussions and suggestions. The research at MIT was funded by NSF Grant DMR-92-01878. The Japanese author (ME) acknowledges the Japan Society for the Promotion of Science for supporting visits to MIT through the U.S.-Japan cooperative research program, and the MIT author (MSD) acknowledges the corresponding support through NSF INT 94-90144. Part of the Japanese work (ME) was supported by a Grant-in Aid for Scientific Research in Priority Area "Carbon Cluster" (Area No. 234/05233214) from the Ministry of Education, Science and Culture, Japan.

\section{REFERENCES}

1. A. Oberlin, M. Endo, and T. Koyama, J. Cryst. Growth 32, 335 (1976).

2. M. Endo, Chemtech 18, 568 (1988).

3. M. Endo, T. Koyama, and Y. Hishiyama, Jpn. J. Appl. Phys. 15, 2073 (1976).

4. M. Endo, A. Oberlin, and T. Koyama, Jpn. J. Appl. Phys. 16, 1519 (1977).

5. T. Koyama, M. Endo, and Y. Hishiyama, Jpn. J. Appl. Phys. 13, 1933 (1974).

6. T. C. Chieu, M. S. Dresselhaus, and M. Endo, Phys. Rev. B 26, 5867 (1982).

7. T.C. Chieu, G. Timp, M. S. Dresselhaus, M. Endo, and A. W. Moore, Phys. Rev. B 27, 3686 (1983).

8. R. Bacon, J. Appl. Phys. 31, 283 (1960).

9. K. Oshida, M. Endo, T. Nakajima, S. L. di Vittorio, M. S. Dresselhaus, and G. Dresselhaus, J. Mater. Res. 8, 512 (1993).

10. M. Endo, A. Oberlin, and T. Koyama, Carbon 14, 133 (1976).

11. M. Endo, Y. Hishiyama, and T. Koyama, J. Phys. D, Appl. Phys. 15, 353 (1982).

12. M Endo, K. Oshida, K. Takeuchi, Y. Sasuda, K. Matsubayashi, and M.S. Dresselhaus, Trans. Inst. Electronics, Information and Communication Eng. J77-C-II, 139 (1994), (in Japanese).

13. S. P. Kelty and C. M. Lieber, J. Phys. Chem. 93, 5983 (1989).

14. H. Saadaoui, J. C. Roux, and S. Flandrois, Carbon 31, 481 (1993). 